\title{
F-responses: a study of frequency, shape and amplitude characteristics in healthy control subjects
}

\author{
SOTIRIA PEIOGLOU-HARMOUSSI, ${ }^{*}$ PETER RW FAWCETT, ${ }^{*}$ DENISE HOWEL,$\dagger$ \\ DAVID D BARWICK*
}

\begin{abstract}
From the Department of Clinical Neurophysiology, Regional Neurological Centre, Newcastle General Hospital, ${ }^{*}$ and the Department of Medical Statistics, University of Newcastle upon Tyne, $\dagger U K$
\end{abstract}

SUMMARY Characteristics of the surface recorded F-response, including frequency, occurrence of identical responses, shape and amplitude have been investigated in the ulnar nerve of control subjects. During a train of 200 stimuli, F-response frequency varied between $50 \%$ and $93 \%$ in different subjects with a mean of $79 \%$. The vast majority of responses $(96.6 \%)$ occurred only once; of those responses which repeated, 89.5\% occurred between 2-5 times, 9\% between 6-10 times and only $1.5 \% 11$ or more times. F-response shape was variable, the majority containing two or more negative peaks. F-response amplitudes tended to be a relatively small proportion of the compound $\mathrm{M}$-response, with median $\mathrm{F} \% \mathrm{M}$ values ranging from $0.8 \%$ to $4 \%$. The data suggest that a large proportion of surface recorded F-responses following supramaximal stimulation are composed of recurrent discharges derived from more than one motor unit.

The basic neurophysiological mechanisms underlying the production of F-responses by antidromic invasion of the anterior horn cell body have been shown by the studies of Renshaw, ${ }^{1}$ Eccles $^{2}$ and Schiller and Stålberg ${ }^{3}$ to be highly complex. The generation of a recurrent discharge may be influenced by a variety of factors, the principal of which is the balance of excitatory and inhibitory post-synaptic potentials on individual anterior horn cells. These factors have a direct effect on the critical phase which is the time required for repolarisation of the axon hillock before reinvasion of this segment by the soma-dendritic spike. ${ }^{3}$

In view of the uncertain conditions surrounding the production of the F-response, knowledge of its frequency in individual motor neurons and the motor neuron pool as a whole would be of value, particularly as these parameters may be altered by diseases which affect the upper and/or lower motor

Present address of Dr S Harmoussi PhD, B. Department of Neurology, Central Hospital, Saint Dimitrios, Salonika, Greece.

Address for reprint requests: Dr PRW Fawcett, Department of Clinical Neurophysiology, Regional Neurological Centre, Newcastle General Hospital, Westgate Road, Newcastle upon Tyne, NE4 6BE, UK

Received 6 November 1984. Accepted 12 January 1985 neurons. This paper presents our findings of F-response frequency in a population of healthy subjects, and will form the basis of control data for comparison with patients presenting with different pathological conditions involving the central and peripheral motor systems.

The study was performed using surface electrodes in order that the results would be available for use in the routine clinical setting. In addition, the F-response shape and amplitude were examined in the large sample obtained during the train of 200 stimuli used, and compared with values obtained in the previously reported study based on 20 F-responses. ${ }^{4}$

\section{Subjects and methods}

The control population consisted of 21 healthy subjects drawn mainly from medical and nursing staff. There were 14 males and seven females with an age range of 30 to 66 years (mean 48 SD 10). All the volunteers were free of cancer, diabetes, intervertebral disc disease and had no neurological symptoms or signs. Informed consent was obtained from each subject.

The subjects reclined on a couch with the arm and hand outstretched and immobilised in the supine position. The skin temperature of the hand was monitored with a thermistor (Light Laboratories, Brighton, Sussex) and maintained at about $34^{\circ} \mathrm{C}$ with a heating lamp. A series of 200 
sequential supra-maximal stimuli was applied to the ulnar nerve at the wrist using bipolar suface electrodes. The stimulus frequency was $1 \mathrm{~Hz}$ with a pulse duration of 0.2 ms. Belly-tendon recordings were made from the hypothenar muscles with surface electrodes, taking care to position the active electrode over the end-plate zone. A sweep speed of $5 \mathrm{~ms}$ per division and sensitivity of $0.2 \mathrm{mV}$ or 0.5 $\mathrm{mV}$ per division were used. The subject was encouraged to relax in order to avoid interfering volitional activity. All the studies were performed on a Medelec MS6 EMG apparatus and the signals were recorded with a fibre-optic recorder using a paper speed of $5 \mathrm{~cm} / \mathrm{s}$ in the raster mode. In order to differentiate responses from background noise, only those deflections which showed a clear deviation from the base-line and had an amplitude of at least $40 \mu \mathrm{V}$ were accepted as F-responses. The latency to onset and peakto-peak amplitudes of each F-response were measured using transparent graticuled graph paper.

\section{Identical F-responses}

F-responses were considered to be identical on the basis of their latencies $( \pm 0.1 \mathrm{~ms})$ and shapes. When two or more F-responses were suspected of being identical, the first was copied on to tracing paper and this copy was superimposed on subsequent similar responses. If superimposition proved their shapes to be virtually identical, they were considered to be the same response.

\section{F-response shape}

The shape of the F-response was assessed in terms of the number of negative peaks which achieved an amplitude of at least $10 \mu \mathrm{V}$. The first $100 \mathrm{~F}$-responses were examined in this manner.

The frequency of valid F-responses together with their latency, amplitude and number of identical responses were presented for analysis. In each subject the following parameters were determined: (1) Overall F-response frequency. This was defined as the percentage of 200 stimuli which produced an F-response. (2) Frequency of identical responses. Because of the variable number of responses occurring during the train of 200 stimuli in different subjects, the repetition frequency of individual responses was determined as shown in the following example. Of the 200 stimuli administered, the number of resultant F-response shapes which occurred singly was 130 , and those which occurred more than once were as follows:

Wave A occurred $\times 3$

$$
\begin{array}{lll}
\text { B }, & \times 3 \\
\text { C }, & \times 4 \\
\text { D } & \times & \times 8 \\
\text { E }, & \times 15
\end{array}
$$

Total number of F-responses $=163$

Total number of F-response shapes $=135$

These results can be tabulated as follows:

No of

occurrences

$$
12-5 \quad 6-10 \quad 11-15 \quad 16-20
$$

Proportion of

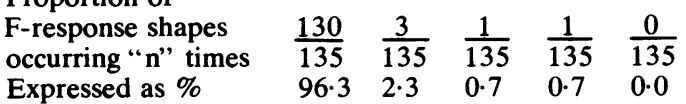

Expressed as \%

The percentages of different F-response shapes occurring (a) singly and (b) two or more times were determined and the frequency with which each repeating wave shape occurred per 200 stimuli was assessed. (3) F-response amplitude. The median and maximum values of the individual amplitudes expressed as a percentage of the M-response (F\%M) were determined. Median rather than mean values were preferred because there was a tendency for the distribution of the amplitudes to be positively skewed.

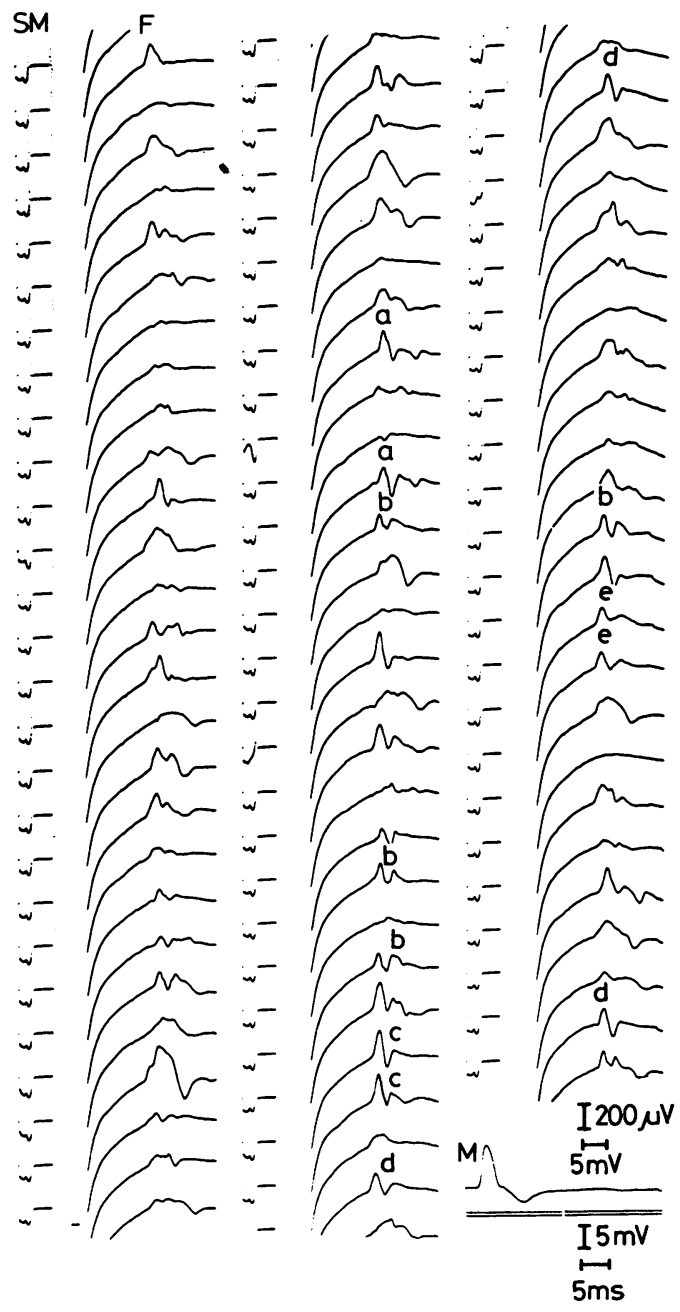

Fig 1 Representative section of a recording of sequential F-responses obtained during a train of 200 supramaximal stimuli to the ulnar nerve at the wrist in a 49-year-old male control subject. Recordings made from the hypothenar muscles of the non-dominant hand. Note the typical variation in latency and configuration of the $F$-responses, and also the occasional identical responses $(a, b, c, d, e, f)$. $S=$ Stimulus, $M=$ Direct compound muscle action potential, $F=F$-response. 
Table 1 F-response frequency during a train of 200 stimuli in the ulnar nerve in controls

\begin{tabular}{|c|c|c|c|}
\hline & Mean $(S D)$ & Minimum & Maximum \\
\hline $\begin{array}{l}\text { F-response frequency } \\
\text { Mean percentage of } \\
\text { F-response shapes } \\
\text { which repeat }\end{array}$ & $79 \cdot 1(10)$ & 50 & 93 \\
\hline
\end{tabular}

Table 2 Frequency of identical F-responses occurring during a train of 200 stimuli in the ulnar nerve in controls

\begin{tabular}{llllll}
\hline & $N$ & 1 & $2-5$ & $6-10$ & $11-15$ \\
\hline $\begin{array}{c}\text { Mean percentage of } \\
\begin{array}{c}\text { F-response shapes } \\
\text { occurring N times }\end{array}\end{array}$ & 96.6 & 2.96 & 0.34 & 0.07 \\
\hline
\end{tabular}

\section{Results}

Satisfactory recordings were obtained in all 21 control subjects. Part of a 200 train sequence recorded from a control subject is illustrated in fig 1 . In this particular example, F-responses were judged to have occurred in all but one of the traces shown. The majority of responses occurred only once, but a small proportion occurred two or more times. The data concerning F-response frequency and frequency of identical responses in all of the control subjects are summarised in tables 1 and 2 .

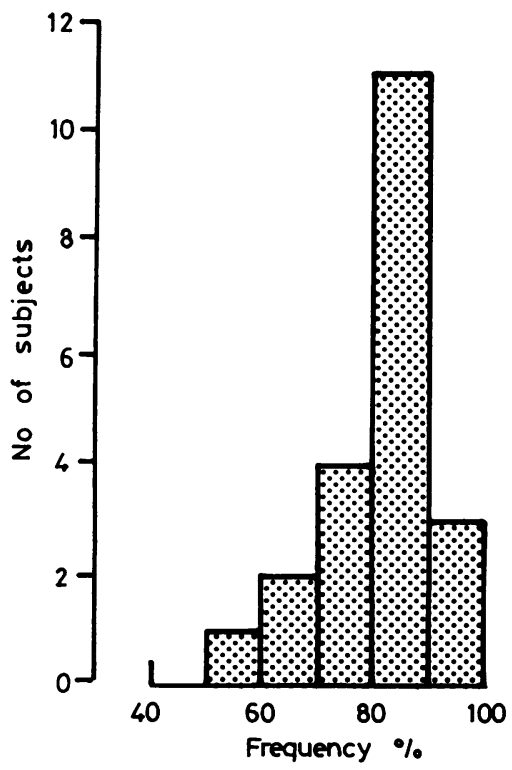

Fig 2 Histogram of the distribution of F-response frequencies in a group of control subjects.

\section{F-response frequency}

During a train of 200 stimuli at a rate of $1 \mathrm{~Hz}$, the frequency of F-responses arising from the whole muscle ranged from $50 \%$ to $93 \%$ with a mean of $79 \%$. The distribution of frequency values in the control group is shown in fig 2 , from which it can be seen that in the majority of cases F-responses occurred following $70 \%$ to $90 \%$ of stimuli, indicating a relatively high chance of an F-response being produced with each stimulus.

\section{Frequency of identical F-responses}

The vast majority of F-response shapes (mean $96.6 \%$ SD 1.1 ) in each control subject occurred only once during the train of 200 stimuli, while between $1.1 \%$ and $5.4 \%$ (mean $3.4 \%$ ) of responses occurred

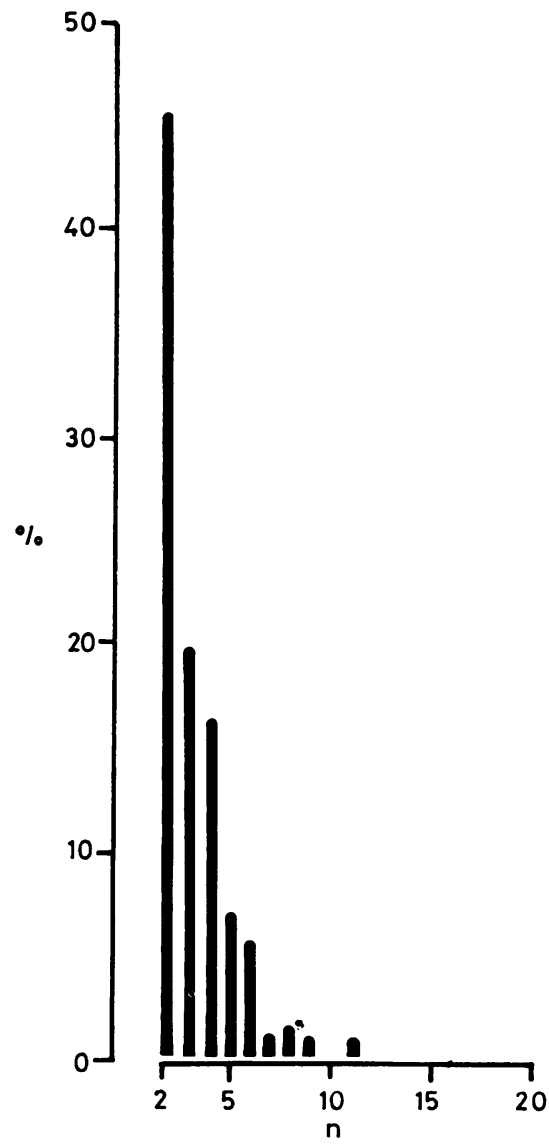

Fig 3 Frequency with which each repeating $F$-response occurred during a train of 200 stimuli in a group of control subjects. Ordinate, percentage of repeating $F$-responses. Abscissa, number of times each repeating response occurred. 


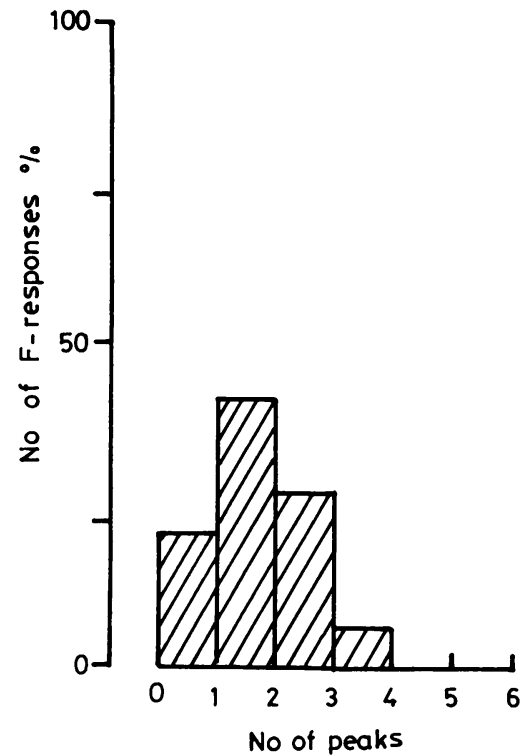

Fig 4 Histogram of the number of negative peaks in $F$-responses recorded from a group of control subjects.

two or more times. The frequency with which each repeating response occurred varied from subject to subject, those occurring 2 to 5 times contributing between $50 \%$ to $100 \%$ of all "repeaters" per subject (mean $89.5 \%$ ), while those occuring 6 to 10 times accounted for $9 \%$. Only two subjects had identical responses which occurred over 10 times (11 times in each case). The average profile of identical responses is presented in fig 3 .

\section{F-response shape}

There was a considerable variation in F-response shape in the control subjects, as illustrated in fig 1. The distribution of the number of negative peaks observed in a total of 1800 responses is presented in fig 4, which shows that a large proportion of responses had a relatively complex configuration with two or three peaks. This was the case for the identical responses as well as for most of the single responses.

Table 3 Summary of F-response amplitude values obtained during a train of 200 stimuli in the ulnar nerve in controls

\begin{tabular}{|c|c|c|c|c|}
\hline & Mean & Median & Minimum & Maximum \\
\hline \multirow{2}{*}{$\begin{array}{l}\text { Median F\%M amplitud } \\
\text { Maximum F\%M } \\
\text { amplitude }\end{array}$} & $1 \cdot 8$ & $1 \cdot 7$ & $0 \cdot 8$ & $4 \cdot 0$ \\
\hline & 5.9 & $5 \cdot 3$ & $2 \cdot 7$ & $11 \cdot 3$ \\
\hline
\end{tabular}

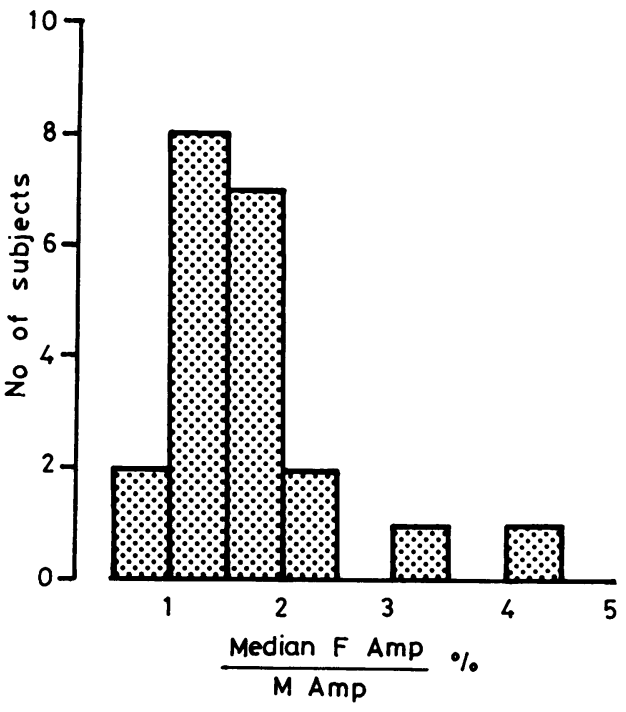

Fig 5 Histogram of the median values of the F-response amplitudes from each subject expressed as a percentage of the M-response amplitude (F\%M).

\section{F-response amplitude}

In each subject the distribution of individual F-response amplitudes expressed as a percentage of the $\mathrm{M}$-response $(\mathrm{F} \% \mathrm{M})$ tended to be positively skewed. Median values were therefore obtained in each case and these are presented in fig 5. The

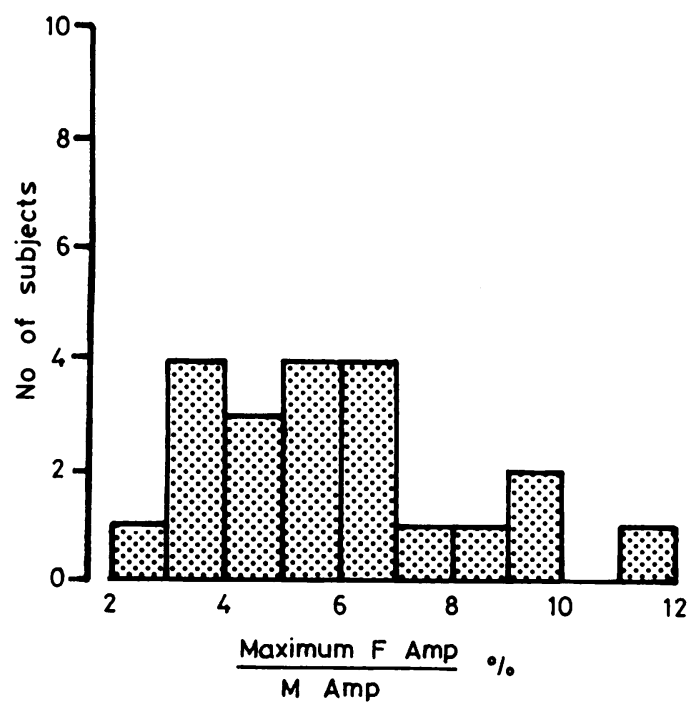

Fig 6 Histogram of the maximum F-response amplitude seen in each subject, expressed as a percentage of the $M$-response amplitude (Max F\%M). 
median values also showed a positively skewed distribution, with values ranging from $0.8 \%$ to $4 \%$ and a median of $1.7 \%$. The maximum F-response amplitude (Max F\%M) obtained in each subject varied between $2.7 \%$ and $11.3 \%$ (median $5.3 \%$ ), (table 3 and fig 6).

\section{Discussion}

While the measurement of F-response latency has proved to be of considerable value in the investigation of patients with polyneuropathies or nerve entrapment syndromes, less attention has been paid to other aspects of the response, in particular F-response frequency. Schiller and Stålberg, ${ }^{3}$ employing single fibre EMG found that only $41 \%$ of individual motor units produced an $\mathrm{F}$ discharge during a series of 200 stimuli, although all motor units would do so during longer stimulus trains. Thirtyfive per cent of the motor units examined produced between 1 to 5 responses per 200 stimuli, while much smaller proportions of $4 \%$ and $2 \%$ gave rise to 6 to 10 and 11 to 15 responses respectively. Yates and Brown, ${ }^{5}$ using surface recording techniques, also found that a relatively small proportion (23\%) of motor units in control subjects generated an F-response in the course of 100 stimuli. Furthermore there was a considerable variation among different subjects, one producing no recurrent discharges in nine motor units studied, while $45 \%$ of units did so in another subject.

The present study has also shown quite a marked variation in F-response frequency arising from the motor unit pool as a whole, with values ranging from $50 \%$ to just over $90 \%$ during trains of 200 stimuli in different control subjects. Examination of the shape and latency characteristics of the individual responses revealed that the vast majority occurred only once, those responses occurring two or more times accounting for between $1 \cdot 1 \%$ to $5 \cdot 4 \%$ of the total number of different responses.

Using the motor unit count method, ${ }^{67}$ the number of motor units within the hypothenar muscles has been estimated to be of the order of 300 to 380 (range approximately 125 to 500).${ }^{89}$ On the basis of the single unit F-discharge frequencies observed by Schiller and Stålberg, ${ }^{3}$ a motor unit pool of this size might be expected to generate a minimum of about 150 and an average of about 370 late responses from individual motor units. This estimated frequency of motor unit late responses clearly exceeds the observed surface recorded F-response frequencies obtained in our control subjects. One of the reasons for this discrepancy may be that low amplitude responses may not have been clearly dentified from background noise leading to the exclusion of responses from small motor units. Furthermore the use of surface recording methods is less precise than the SFEMG technique and consequently it is not possible to ascribe F-responses to individual motor units. Thus an unknown proportion of F-responses may have resulted from the summation of two or more different motor units which have simultaneously generated late responses. This factor would also result in an underestimate of the number of repeating F-responses. Nevertheless it is encouraging to note that the rates at which individual responses recurred during the 200 stimulus train correspond reasonably closely with the SFEMG findings of Schiller and Stålberg. ${ }^{3}$

These observations suggest that whereas a large proportion of motor units are capable of generating recurrent discharges, the conditions that prevail at the level of the cell body at a given instant are only rarely favourable for their development in any one motor neuron. This is of interest with regard to the well described size principle ${ }^{9-12}$ concerning the relationship between motor neuron size and order of recruitment during voluntary and reflex activity. It would thus appear that cell size has little influence on the production of an F-response, and this agrees with the findings of Kimura and colleagues ${ }^{13}$ that F-responses are generated in both large and small motor neurons alike. It is also clear from the observations of Schiller and Stålberg ${ }^{3}$ on the occurrence of F-responses during prolonged trains of stimuli that they are not random events, since they tend to occur in clusters, suggesting that there are slow fluctuations in the balance of factors underlying their generation.

The range of F-response amplitudes in the present large sample compares closely with the values obtained in a smaller sample of $20,{ }^{4}$ confirming that the latter sample size is probably adequate for routine clinical use. Expressed as a percentage of the $\mathrm{M}$-response, the F-amplitudes were larger than the amplitudes of surface recorded values produced by the earliest recruited motor units following direct graded stimulation of the ulnar nerve. ${ }^{7}$ This is in accordance with the findings of Feasby and Brown ${ }^{14}$ who suggested that the large F-responses were derived from the larger, high threshold, motor units. While this may be true for some of the responses, summation of recurrent discharges from two or more motor units will also account for a number of the larger amplitudes.

Another characteristic of the F-response which has received little attention is the shape. In the present study there was considerable variation in the configuration of the responses within each control subject, with the majority of responses having two or three identifiable negative peaks. The main fac- 
tors determining the shape of the waveform are likely to be the position of the active recording electrode with regard to the end-plate region of the motor unit, the degree of temporal synchronisation of the arrival of nerve impulses in the terminal branches and subsequently the muscle fibres, and the possible concurrent discharge of two or more separate motor units. As all of these factors may be altered by pathological changes involving the upper and lower motor neurons, examination of the F-response shape might be expected to be of value in the assessment of a variety of conditions affecting the motor system.

In conclusion this paper presents a detailed account of some of the more recently recognised F-response parameters in a population of healthy subjects, and forms the basis of control data to be used in the study of patients with diseases of both upper and lower motor neurons.

Dr S Peioglou-Harmoussi was supported by a Greek State Scholarship Fund (IKY).

\section{References}

' Renshaw B. Influence of discharge of motor neurones upon excitation of neighbouring motorneurones. $J$ Neurophysiol 1941;4:167-83.

${ }^{2}$ Eccles JC. The central action of antidromic impulses in motor nerve fibres. Pfugers Arch 1955;260:380-415.

${ }^{3}$ Schiller HH, Stålberg E. F responses studied with single fibre EMG in normal subjects and spastic patients. $J$
Neurol Neurosurg Psychiatry 1978;41:45-53.

${ }^{4}$ Peioglou-Harmoussi S, Howel D, Fawcett PRW, Barwick DD. F-response behaviour in a control population. J Neurol Neurosurg Psychiatry 1985;48: 1152-58.

5 Yates SK, Brown WF. Characteristics of the F response: a single motor unit study. J Neurol Neurosurg Psychiatry 1979;42:161-70.

- McComas AJ, Fawcett PRW, Campbell MJ, Sica REP. Electrophysiological estimation of the number of motor units within a human muscle. J Neurol Neurosurg Psychiatry 1971;34:121-31.

${ }^{7}$ Sica REP, McComas AJ, Upton ARM, Longmire D. Motor unit estimations in small muscles of the hand. $J$ Neurol Neurosurg Psychiatry 1974;37:55-67.

${ }^{8}$ Milner-Brown HS, Brown WF. New methods of estimating the number of motor units in a muscle. $J$ Neurol Neurosurg Psychiatry 1976;39:258-65.

${ }^{9}$ Henneman E, Somjen G, Carpenter DO. Functional significance of cell size in spinal motorneurones. $J$ Neurophysiol 1965;28:560-80.

${ }^{10}$ Hannerz J. Discharge properties of motor units in relation to recruitment order in voluntary contraction. Acta Physiol Scand 1974;91:374-84.

"Henneman E, Shahani BT, Young RR. Voluntary control of human motor units. Neurology (Minneap) 1975;25:368,

${ }^{12}$ Milner-Brown HS, Stein RB, Yemm R. The orderly recruitment of human motor units during voluntary $\square$ contractions. J Physiol (Lond) 1973;230:359-70.

${ }^{13}$ Kimura J, Yanagisawa H, Yamada T, Mitsudome A, Sasaki H, Kimura A. Is the $F$ wave elicited in a select $\mathbb{D}$ group of motorneurones? Muscle Nerve 1984;7:3929.

${ }^{14}$ Feasby TE, Brown WE. Variation of motor unit size in $\bigcirc$ the human extensor digitorum brevis and thenar muscles. J Neurol Neurosurg Psychiatry 1974;37:916-26. 